\title{
Screening Ionic Liquids by COSMO-RS Method for Preparation of Antibacterial Cellulose Fibers
}

\author{
Xinxin Li, ${ }^{a, b}$ Hongshuai Gao, ${ }^{* b, c}$ Le Zhou, ${ }^{c}$ and Yi Nie*a,b,c
}

a School of Chemical Engineering, Zhengzhou University, Zhengzhou 450000, China.

b Zhengzhou Institute of Emerging Industrial Technology, Zhengzhou 450000, China.

${ }^{c}$ Beijing Key Laboratory of Ionic Liquids Clean Process, CAS State Key Laboratory of

Multiphase Complex Systems, CAS Key Laboratory of Green Process and Engineering, Institute of Process Engineering, Chinese Academy of Sciences, Beijing 100190, China.

Corresponding Author: E-mail: hsgao@ipe.ac.cn, ynie@ipe.ac.cn

Number of Pages: 9

Number of Tables: 7

Number of Figures: 5 
1. Structures of cations and anions

Table S1. Cations studied in this work

\begin{tabular}{lll}
\hline No. & Name of cation & Acronym \\
\end{tabular}

Emim

Amim

1-R-3- Bmim

$1-6$

Methylimidazolium HOEtmim<smiles>[R][n+]1ccn(C)c1</smiles>

EtOMmim

AOPmim

Epy

Apy

7-10 N - R- Pyridinium

HOEtpy<smiles>[R][n+]1ccccc1</smiles>

EtOMpy

$\mathrm{Ch}$<smiles>C[N+](C)(C)CCO</smiles>

11-12 Choline

$\mathrm{ACh}$<smiles>CC(=O)OCC[N+](C)(C)C</smiles> 
1-R-1-

13

Table S2. Anions studied in this work

\begin{tabular}{|c|c|c|c|}
\hline NO. & Name of cation & Acronym & Structure \\
\hline 1 & $\mathrm{Ac}^{-}$ & Acetate & \\
\hline 2 & $\mathrm{HCOO}^{-}$ & Formate & \\
\hline 3 & $\mathrm{BEN}^{-}$ & Benzoate & \\
\hline 4 & $\operatorname{Dec}^{-}$ & Decanoate & \\
\hline 5 & $\mathrm{Cl}^{-}$ & Chloride & $\mathrm{Cl}^{-}$ \\
\hline 6 & $\mathrm{Br}^{-}$ & Bromide & $\mathrm{Br}^{-}$ \\
\hline 7 & $\mathrm{DEP}^{-}$ & Diethylphosphate & \\
\hline
\end{tabular}




\begin{tabular}{|c|c|c|c|}
\hline 8 & $\mathrm{DBP}^{-}$ & Dibutylphosphate & $-0^{\prime} \mathrm{O}_{\mathrm{O}}^{\mathrm{P}}-\mathrm{O}$ \\
\hline 9 & $\mathrm{DMPO}_{4}^{-}$ & Dimethylphosphate & \\
\hline 10 & $\mathrm{BuSO}_{4}{ }^{-}$ & Butylsulfate & $\mathrm{O}=\begin{array}{c}\mathrm{O}^{-} \\
\mathrm{S}_{1} \\
\mathrm{O}\end{array}$ \\
\hline 11 & $\mathrm{HSO}_{4}^{-}$ & Hydrogensulfate & $\mathrm{O}=\stackrel{\substack{\mathrm{O}_{\mathrm{S}}^{-} \\
\stackrel{\mathrm{O}}{\mathrm{O}} \mathrm{H}}}{\mathrm{O}}$ \\
\hline 12 & $\mathrm{MeSO}_{4}^{-}$ & Methoxyethylsulfate & $\begin{array}{c}\mathrm{O}_{1}^{-} \\
\mathrm{I} \\
\mathrm{S} \\
\mathrm{O} \\
\mathrm{O}\end{array}$ \\
\hline 13 & $\mathrm{TOS}^{-}$ & Toluene-4-sulfonate & O-S \\
\hline 14 & $\mathrm{ClO}_{4}^{-}$ & Perchlorate & $\mathrm{O}=\stackrel{\substack{\mathrm{I} \\
\mathrm{O}^{-}}}{-}=0$ \\
\hline 15 & $\mathrm{PF}_{6}^{-}$ & Hexafluorophosphate & $\underset{F}{F_{X}=-P_{F}^{\prime}-F}$ \\
\hline
\end{tabular}

\section{Synthesis and characterization of ILs}

\subsection{NMR analysis of five synthesis ILs}

[Emim]DEP: ${ }^{1} \mathrm{H}$ NMR (d ${ }_{6}$-DMSO): $9.27(\mathrm{~s}, 1 \mathrm{H}), 7.79(\mathrm{~s}, 1 \mathrm{H}), 7.71(\mathrm{~s}, 1 \mathrm{H}), 4.18(\mathrm{~s}, 2 \mathrm{H}), 3.85$ (s, $3 \mathrm{H}), 3.59(\mathrm{~s}, 4 \mathrm{H}), 1.42(\mathrm{~s}, 3 \mathrm{H}), 1.05(\mathrm{~s}, 6 \mathrm{H})$. 
[Emim]AC: ${ }^{1} \mathrm{H}$ NMR (d ${ }_{6}$-DMSO): $9.90(\mathrm{~s}, 1 \mathrm{H}), 7.95$ (s, 1H), $7.84(\mathrm{~s}, 1 \mathrm{H}), 4.22(\mathrm{~s}, 2 \mathrm{H}), 3.88(\mathrm{~s}$, $3 \mathrm{H}), 1.62(\mathrm{~s}, 3 \mathrm{H}), 1.39(\mathrm{~s}, 3 \mathrm{H})$.

[Amim]Cl: ${ }^{1} \mathrm{H}$ NMR (d ${ }_{6}$-DMSO): 9.68 (s), 7.91 (s), 7.89 (s), 6.04 (s), 5.31 (s), 4.95 (s), 3.91 (s).

[Bmim]Cl: ${ }^{1} \mathrm{H}$ NMR (d $\left.{ }_{6}-\mathrm{DMSO}\right): 9.38(\mathrm{~s}, 1 \mathrm{H}), 7.83$ (t, 1H), 7.76 (s, 1H), 4.19 (s, 2H), 3.87 (s, $3 \mathrm{H}), 1.77(\mathrm{~s}, 2 \mathrm{H}), 1.28(\mathrm{~s}, 2 \mathrm{H}), 0.90(\mathrm{~s}, 3 \mathrm{H})$.

[Bmim]PF ${ }_{6}:{ }^{1} \mathrm{H}$ NMR (d ${ }_{6}$-DMSO): 9.09 (s, 1H), $7.76(\mathrm{~s}, 1 \mathrm{H}), 7.69$ (s, 1H), $4.16(\mathrm{~s}, 2 \mathrm{H}), 3.85(\mathrm{~S}$, $3 \mathrm{H}), 1.77(\mathrm{~s}, 2 \mathrm{H}), 1.27(\mathrm{~s}, 2 \mathrm{H}), 0.91(\mathrm{~s}, 3 \mathrm{H})$.

\subsection{Water content of synthesis ILs}

Table S3. Water content of five ILs

\begin{tabular}{|c|c|c|c|c|c|}
\hline ILs & {$[$ Emim $] \mathrm{DEP}$} & {$[$ Emim $] \mathrm{Ac}$} & {$[\mathrm{Amim}] \mathrm{Cl}$} & {$[\mathrm{Bmim}] \mathrm{Cl}$} & {$\left[\mathrm{Bmim}^{\mathrm{P}} \mathrm{PF}_{6}\right.$} \\
\hline Water content $(\mathrm{ppm})$ & 735.7 & 848.4 & 798.0 & 835.7 & 628.4 \\
\hline
\end{tabular}

\section{Logarithmic activity coefficients (ln $\gamma)$ prediction by COSMO-RS}

Table S4. Prediction $\ln \gamma$ of thymol by COSMO-RS

\begin{tabular}{|c|c|c|c|c|c|c|c|c|c|c|c|c|c|c|c|}
\hline HOEtpy & -4.12 & -3.74 & -3.12 & -3.08 & -3.33 & -3.09 & -2.81 & -2.40 & -1.77 & -1.38 & -1.13 & -0.95 & -0.24 & 0.48 & 0.63 \\
\hline HOEtmim & -4.27 & -3.93 & -3.30 & -3.24 & -3.46 & -3.25 & -2.97 & -2.60 & -1.90 & -1.56 & -1.24 & -1.07 & -0.37 & 0.39 & 0.57 \\
\hline $\mathrm{Ch}$ & -4.48 & -4.11 & -3.44 & -3.40 & -3.58 & -3.34 & -3.05 & -2.64 & -1.92 & -1.58 & -1.22 & -1.03 & -0.30 & 0.50 & 0.70 \\
\hline $\mathrm{ACh}$ & -4.80 & -4.43 & -3.69 & -3.78 & -3.72 & -3.49 & -3.22 & -2.71 & -2.02 & -1.66 & -1.32 & -1.13 & -0.46 & 0.38 & 0.60 \\
\hline HOEtmpyrr & -4.96 & -4.71 & -4.03 & -3.90 & -4.02 & -3.84 & -3.57 & -3.21 & -2.33 & -2.10 & -1.58 & -1.42 & -0.75 & 0.17 & 0.32 \\
\hline AOPmim & -5.05 & -4.78 & -4.08 & -4.05 & -4.03 & -3.85 & -3.60 & -3.18 & -2.37 & -2.09 & -1.67 & -1.51 & -0.91 & -0.01 & 0.25 \\
\hline Epy & -5.16 & -4.88 & -4.15 & -4.15 & -4.11 & -3.92 & -3.66 & -3.20 & -2.40 & -2.07 & -1.67 & -1.50 & -0.83 & 0.11 & 0.39 \\
\hline EtOMpy & -5.18 & -4.91 & -4.19 & -4.19 & -4.13 & -3.95 & -3.70 & -3.24 & -2.44 & -2.12 & -1.71 & -1.54 & -0.90 & 0.04 & 0.33 \\
\hline Apy & -5.20 & -4.92 & -4.19 & -4.19 & -4.14 & -3.96 & -3.70 & -3.23 & -2.43 & -2.10 & -1.70 & -1.53 & -0.88 & 0.06 & 0.35 \\
\hline EtOMmim & -5.19 & -4.95 & -4.23 & -4.19 & -4.15 & -3.98 & -3.73 & -3.31 & -2.47 & -2.19 & -1.73 & -1.57 & -0.93 & 0.02 & 0.32 \\
\hline Emim & -5.22 & -4.99 & -4.26 & -4.21 & -4.19 & -4.02 & -3.77 & -3.36 & -2.49 & -2.21 & -1.75 & -1.58 & -0.92 & 0.05 & 0.32 \\
\hline Amim & -5.36 & -5.12 & -4.39 & -4.34 & -4.29 & -4.12 & -3.86 & -3.44 & -2.55 & -2.29 & -1.81 & -1.64 & -1.00 & -0.01 & 0.31 \\
\hline Bmim & -5.31 & -5.12 & -4.42 & -4.35 & -4.29 & -4.14 & -3.91 & -3.51 & -2.63 & -2.39 & -1.88 & -1.63 & -1.13 & -0.15 & 0.18 \\
\hline $\begin{array}{l}\text { Cations } \\
\text { Anions }\end{array}$ & Dec & Ac & $\mathrm{CHOO}$ & BEN & DBP & DEP & $\mathrm{DMPO}_{4}$ & $\mathrm{Cl}$ & TOS & $\mathrm{Br}$ & $\mathrm{BuSO}_{4}$ & $\mathrm{MeSO}_{4}$ & $\mathrm{HSO}_{4}$ & $\mathrm{ClO}_{4}$ & $\mathrm{PF}_{6}$ \\
\hline
\end{tabular}


Table S5. Prediction $\ln \gamma$ of quercetin by COSMO-RS

\begin{tabular}{|c|c|c|c|c|c|c|c|c|c|c|c|c|c|c|c|}
\hline HOEtpy & -5.43 & -5.31 & -4.86 & -5.08 & -4.84 & -5.09 & -4.78 & -4.09 & -3.37 & -3.46 & -2.39 & -2.40 & -1.58 & -0.08 & 0.19 \\
\hline HOEtmim & -5.62 & -5.46 & -5.03 & -5.24 & -5.02 & -5.22 & -4.94 & -4.25 & -3.56 & -3.59 & -2.51 & -2.51 & -1.70 & -0.14 & 0.16 \\
\hline $\mathrm{Ch}$ & -5.67 & -5.52 & -5.16 & -5.31 & -5.07 & -5.30 & -4.99 & -4.27 & -3.61 & -3.62 & -2.47 & -2.49 & -1.64 & -0.03 & 0.28 \\
\hline HOEtmpyrr & -6.01 & -5.72 & -5.65 & -5.64 & -5.43 & -5.57 & -5.36 & -4.52 & -4.08 & -3.96 & -2.81 & -2.79 & -2.01 & -0.28 & 0.12 \\
\hline Emim & -6.69 & -6.39 & -5.96 & -6.12 & -6.01 & -6.05 & -5.81 & -5.05 & -4.24 & -4.19 & -2.97 & -2.96 & -2.16 & -0.36 & 0.04 \\
\hline EtOMmim & -6.72 & -6.41 & -5.99 & -6.12 & -6.05 & -6.06 & -5.83 & -5.08 & -4.26 & -4.20 & -2.97 & -2.96 & -2.20 & -0.38 & 0.04 \\
\hline Epy & -6.72 & -6.45 & -5.93 & -6.12 & -6.02 & -6.07 & -5.80 & -5.06 & -4.15 & -4.15 & -2.91 & -2.91 & -2.09 & -0.31 & 0.08 \\
\hline Apy & -6.74 & -6.46 & -5.97 & -6.14 & $\begin{array}{l}-6.04 \\
\end{array}$ & -6.09 & -5.82 & -5.07 & -4.18 & -4.17 & -2.93 & -2.93 & $\begin{array}{l}-2.13 \\
\end{array}$ & -0.34 & 0.04 \\
\hline EtOMpy & -6.76 & -6.46 & -5.99 & -6.14 & -6.07 & -6.08 & -5.83 & -5.10 & -4.21 & -4.18 & -2.94 & -2.93 & -2.16 & -0.35 & 0.05 \\
\hline AOPmim & -6.76 & -6.47 & -5.98 & -6.13 & -6.08 & -6.07 & -5.83 & -5.13 & -4.26 & -4.20 & -2.97 & -2.97 & -2.25 & -0.43 & -0.01 \\
\hline $\mathrm{ACh}$ & -6.66 & -6.47 & -5.75 & -5.98 & -5.92 & -5.98 & -5.64 & -5.03 & -3.95 & -3.97 & -2.68 & -2.71 & -1.90 & -0.13 & 0.25 \\
\hline Bmim & -6.73 & -6.36 & -6.05 & -6.15 & -6.08 & -6.05 & -5.87 & -5.09 & -4.36 & -4.26 & -2.82 & -3.02 & -2.31 & -0.49 & -0.06 \\
\hline Amim & -6.83 & -6.51 & -6.12 & -6.23 & -6.14 & -6.17 & -5.93 & -5.15 & -4.35 & -4.28 & -3.03 & -3.02 & -2.24 & -0.40 & 0.02 \\
\hline $\begin{array}{l}\text { Cations } \\
\text { Anions }\end{array}$ & Ac & Dec & $\mathrm{Cl}$ & DEP & $\mathrm{CHOO}$ & DBP & $\mathrm{DMPO}_{4}$ & BEN & $\mathrm{Br}$ & TOS & $\mathrm{MeSO}_{4}$ & $\mathrm{BuSO}_{4}$ & $\mathrm{HSO}_{4}$ & $\mathrm{ClO}_{4}$ & $\mathrm{PF}_{6}$ \\
\hline
\end{tabular}

Table S6. Prediction ln $\gamma$ of aloe-emodin by COSMO-RS

\begin{tabular}{|c|c|c|c|c|c|c|c|c|c|c|c|c|c|c|c|}
\hline HOEtpy & -3.96 & -3.71 & -3.30 & -3.08 & -2.81 & -3.03 & -2.88 & -2.77 & -1.49 & -1.57 & -0.82 & -0.78 & -0.35 & 0.21 & 0.15 \\
\hline HOEtmim & -4.11 & -3.82 & -3.45 & -3.19 & -2.95 & -3.13 & -3.00 & -2.89 & -1.63 & -1.66 & -0.89 & -0.86 & $\begin{array}{l}-0.44 \\
\end{array}$ & 0.18 & 0.16 \\
\hline $\mathrm{Ch}$ & -4.22 & -3.93 & -3.53 & -3.28 & -3.06 & -3.22 & -3.06 & -2.95 & -1.67 & -1.68 & -0.87 & -0.82 & -0.38 & 0.28 & 0.27 \\
\hline HOEtmpyrr & -4.50 & -4.08 & -3.84 & -3.51 & -3.43 & -3.40 & -3.33 & -3.14 & -2.01 & -1.89 & -1.05 & -1.03 & -0.64 & 0.11 & 0.18 \\
\hline AOPmim & -4.95 & -4.55 & -4.19 & -3.77 & -3.51 & -3.67 & -3.57 & -3.49 & -2.01 & -1.95 & -1.07 & -1.05 & -0.72 & 0.11 & 0.21 \\
\hline$\overline{\mathrm{ACh}}$ & -4.95 & -4.64 & -4.13 & -3.72 & -3.36 & -3.67 & -3.49 & -3.48 & -1.83 & -1.84 & -0.93 & -0.89 & -0.49 & 0.32 & 0.38 \\
\hline EtOMmim & -5.04 & -4.62 & -4.28 & -3.85 & -3.61 & -3.74 & -3.66 & -3.56 & -2.08 & -2.02 & -1.12 & -1.11 & -0.74 & 0.08 & 0.16 \\
\hline Bmim & -5.04 & -4.56 & -4.30 & -3.86 & -3.64 & -3.72 & -3.68 & -3.56 & -2.14 & -2.05 & -1.15 & -1.10 & -0.82 & -0.01 & 0.08 \\
\hline Emim & -5.06 & -4.64 & -4.30 & -3.89 & -3.64 & -3.77 & -3.69 & -3.58 & -2.11 & -2.05 & -1.16 & -1.14 & -0.75 & 0.07 & 0.14 \\
\hline EtOMpy & -5.08 & -4.67 & -4.30 & -3.87 & -3.59 & -3.77 & -3.67 & -3.58 & -2.03 & -2.01 & -1.11 & -1.09 & -0.72 & 0.09 & 0.16 \\
\hline Apy & -5.09 & -4.70 & -4.30 & -3.89 & -3.59 & -3.79 & -3.67 & -3.58 & -2.02 & -2.02 & -1.12 & -1.10 & -0.71 & 0.09 & 0.14 \\
\hline Epy & -5.09 & -4.70 & $\begin{array}{l}-4.29 \\
\end{array}$ & -3.89 & -3.59 & -3.79 & -3.67 & -3.59 & -2.02 & -2.02 & -1.12 & -1.10 & -0.69 & 0.10 & 0.16 \\
\hline Amim & -5.17 & -4.73 & -4.39 & -3.96 & -3.72 & -3.85 & -3.76 & -3.64 & -2.16 & -2.09 & -1.18 & -1.16 & -0.78 & 0.05 & $\overline{0.14}$ \\
\hline $\begin{array}{l}\text { Cations } \\
\text { Anions }\end{array}$ & Ac & Dec & $\mathrm{CHOO}$ & DEP & $\mathrm{Cl}$ & DBP & DMPO4 & BEN & $\mathrm{Br}$ & TOS & $\mathrm{BuSO}_{4}$ & $\mathrm{MeSO}_{4}$ & $\mathrm{HSO}_{4}$ & $\mathrm{ClO}_{4}$ & $\mathrm{PF}_{6}$ \\
\hline
\end{tabular}

\section{Recovery and utilization of ionic liquids}




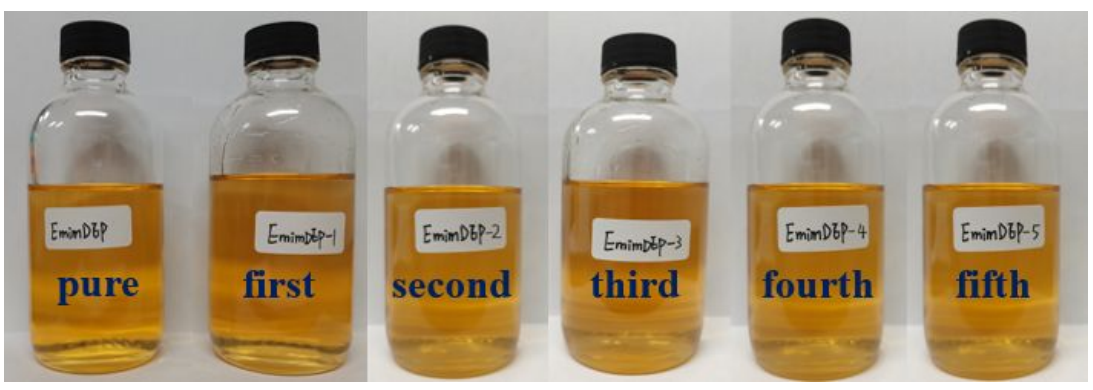

Figure S1. The picture of [Emim]DEP after five recycling

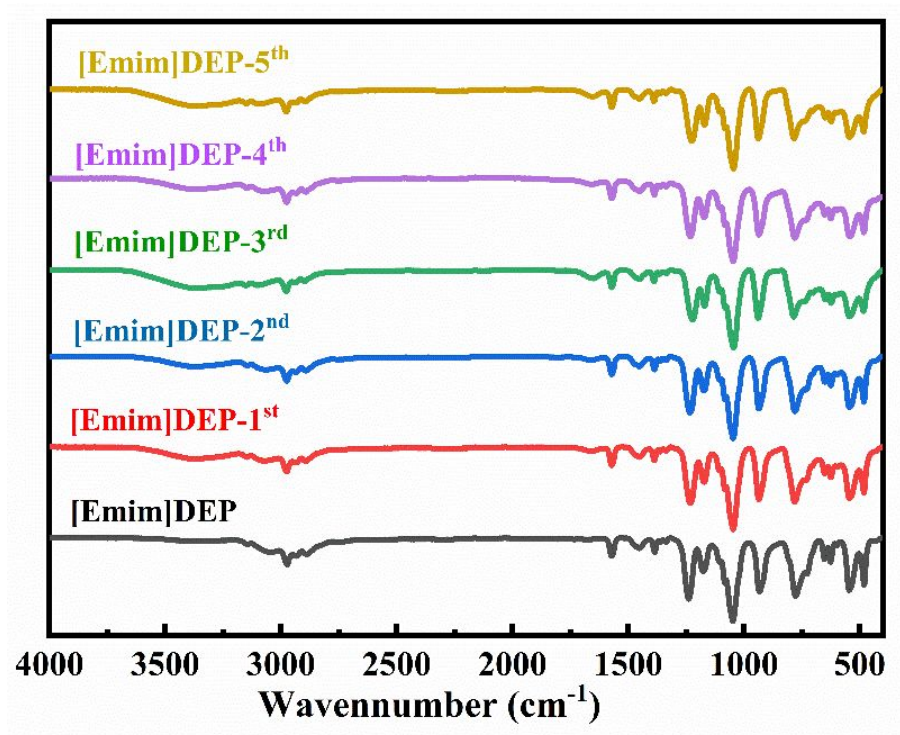

Figure S2. FT-IR spectra of [Emim]DEP after five recycling

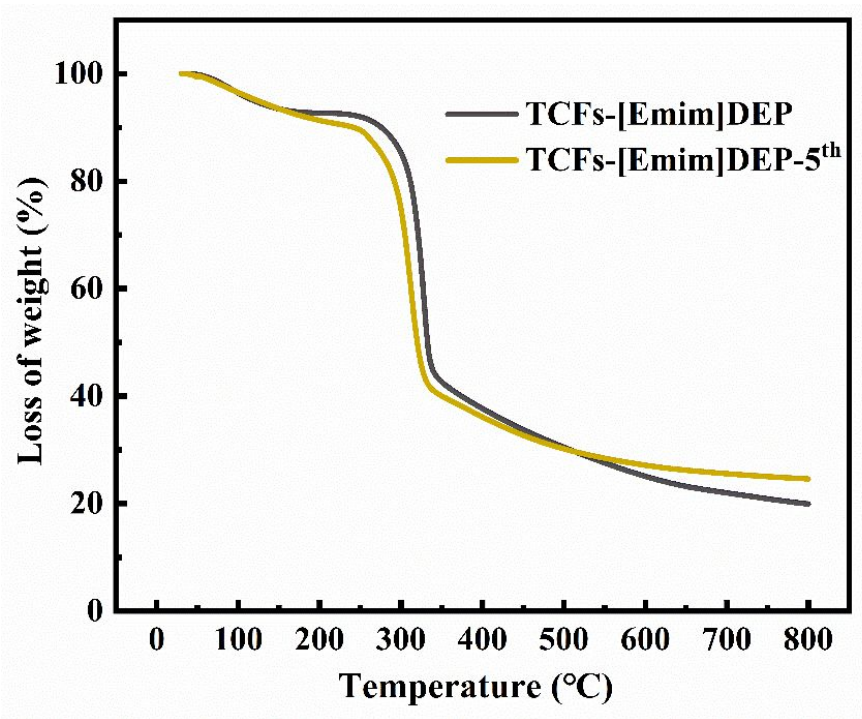

Figure S3. TGA of antibacterial cellulose fibers that prepared by recycled IL and fresh IL 


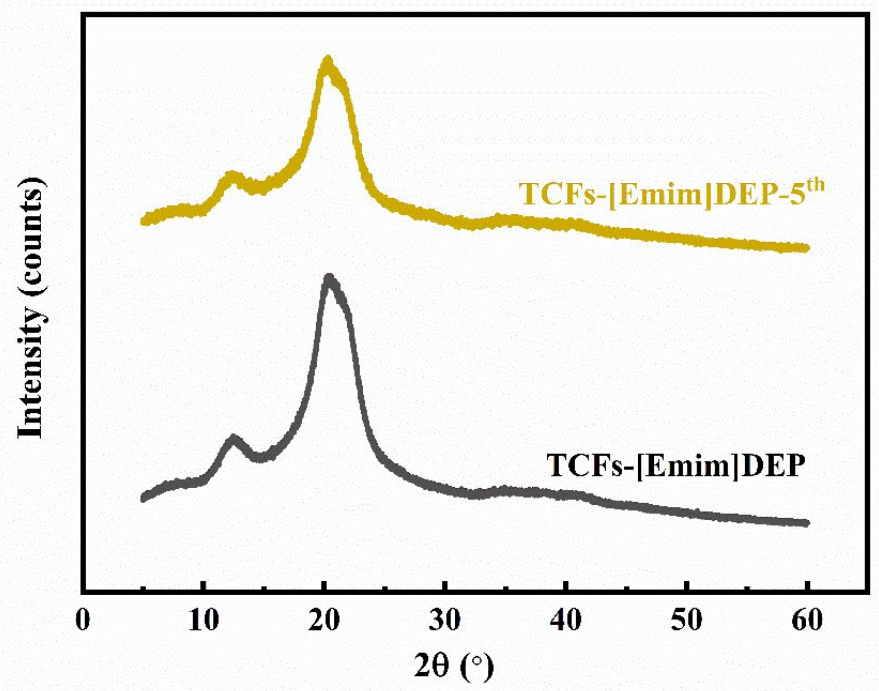

Figure S4. XRD of antibacterial cellulose fibers that prepared by recycled IL and fresh IL

\section{Mechanical property of antibacterial cellulose fibers.}

Table S7. Mechanical property of antibacterial cellulose fibers

\begin{tabular}{|c|c|c|c|c|}
\hline Fiber & Diameter $(\mathrm{mm})$ & Breaking strength $(\mathrm{MPa})$ & Breaking elongation (\%) & Breaking force (N) \\
\hline CFs & 0.0703 & 478.3 & 13.7 & 1.81 \\
\hline QCFs & 0.0686 & 543.1 & 13.8 & 2.01 \\
\hline TCFs & 0.0693 & 556.5 & 14.1 & 2.12 \\
\hline ACFs & 0.0682 & 580.8 & 13.8 & 2.24 \\
\hline
\end{tabular}

\section{The final retention rate of antibacterial agents.}

The concentration of thymol was $40 \mathrm{mg} / \mathrm{mL}, 20 \mathrm{mg} / \mathrm{mL}, 10 \mathrm{mg} / \mathrm{mL}, 8 \mathrm{mg} / \mathrm{mL}, 5 \mathrm{mg} / \mathrm{mL}, 4$ $\mathrm{mg} / \mathrm{mL}$ and $1 \mathrm{mg} / \mathrm{mL}$ using anhydrous ethanol by gradient dilution method. And the absorbance of these solutions was measured by uv spectrophotometer at $300 \mathrm{~nm}$ and the standard curve of thymol was plotted. Then, take the chopped fibers and soak them in anhydrous ethanol. After the migration of antibacterial agent reached the maximum value, the soaking solution was filtered and the absorbance was measured by ultraviolet spectrophotometer. After calculation, the final retention rate of thymol in $25 \%$ TCFs was $12.7 \%$. 


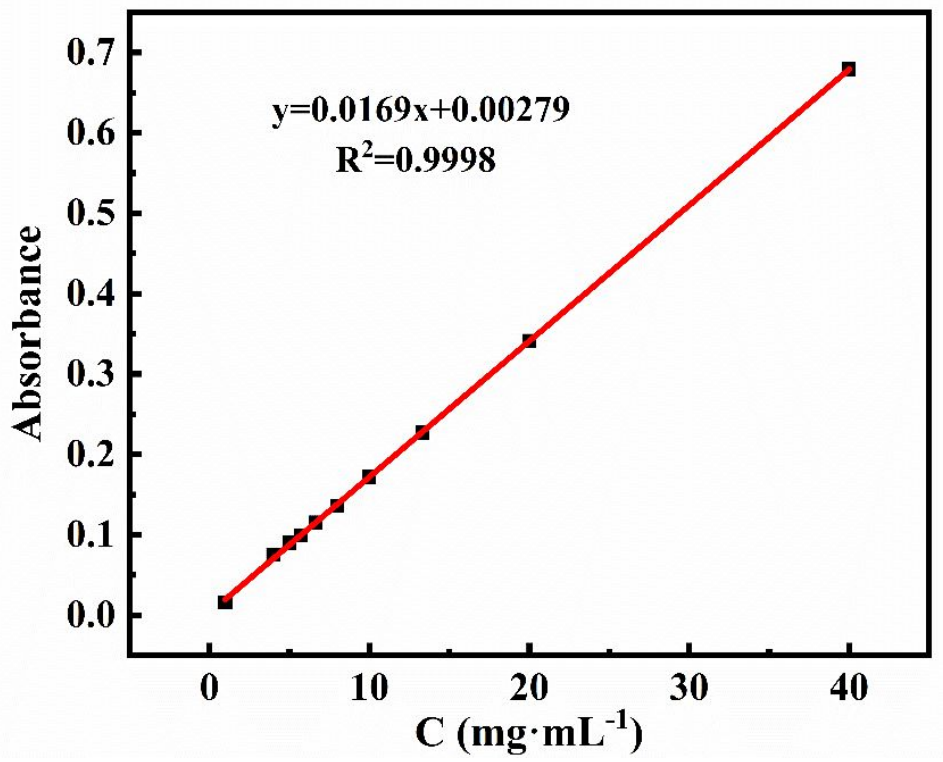

Figure S5. Concentration-absorbance standard curve of thymol at $300 \mathrm{~nm}$ 